\title{
Heparin-Related Major Bleeding in Covid-19-Positive Patient: Perspective from the Outbreak
}

\author{
Pierleone Lucatelli ${ }^{1}$ (I) $\cdot$ Gianluca De Rubeis $^{1} \cdot$ Michele Citone $^{2} \cdot$ Nicola Maria Lucarelli $^{3}$. \\ Valerio Pasqualini ${ }^{4} \cdot$ Mauro Sturiale $^{5} \cdot$ Silvia Giuliani $^{6} \cdot$ Marzia Rosati $^{7}$. \\ Claudio Ceccherini ${ }^{8}$ - Mario Corona ${ }^{1}$ - Cristina Mosconi ${ }^{9}$ Alice Utili ${ }^{10}$. \\ Renato Argirò ${ }^{11}$
}

Received: 28 April 2020/Accepted: 15 May 2020/Published online: 28 May 2020

(C) Springer Science+Business Media, LLC, part of Springer Nature and the Cardiovascular and Interventional Radiological Society of Europe (CIRSE) 2020

\section{To the Editor}

The Covid-19 pandemic, due to its rapid spread [1], is significant straining the various national health systems, especially due to the number of patients requiring longterm support in intensive care unit (ICU). Thus, governments have started massive healthcare unit expansion, supplying medical equipment and personal protective equipment as well as creating and/or converting existing buildings into Covid dedicated field hospitals. These facilities have limited infrastructure, specifically no ability to perform complex imaged guided interventions.

The principal cause of death is acute respiratory insufficiency although concern exists over the concomitant coagulation disorder that can precipitate disseminated intravascular coagulation (DIC) [2]. In light of this, heparin therapy has been introduced recently as an adjuvant
Pierleone Lucatelli

Pierleone.lucatelli@gmail.com

Gianluca De Rubeis

derubeis.gianluca@gmail.com

Michele Citone

michele.citone@gmail.com

Nicola Maria Lucarelli

lucarelli.nico@gmail.com

Valerio Pasqualini

valeriopasqualini@yahoo.it

Mauro Sturiale

sturialem@gmail.com

Silvia Giuliani

silvia.giuliani63@gmail.com

Marzia Rosati

marzia_rosati@hotmail.com

Claudio Ceccherini

claudiocec@hotmail.it

Mario Corona

mario.corona68@gmail.com

Cristina Mosconi

cristina.mosconi@aosp.bo.it

Alice Utili

alice.utili@hsanmartino.it
Renato Argirò

renato.argiro@gmail.com

1 Department of Radiological Oncological and AnatomoPathological Sciences, Sapienza University of Rome, Viale Regina Elena 324, 00161 Rome, Italy

2 Vascular and Interventional Radiology Department, Careggi University Hospital, Florence, Italy

3 Azienda Ospedaliera-Universitaria Consorziale Policlinico Bari, Bari, Italy

4 Ospedale San Filippo Neri, Rome, Italy

5 Policlinico Militare Celio, Rome, Italy

6 Radiologia Interventistica, Ospedale San Camillo, Rome, Italy

7 AOU Ospedali Riuniti, Ancona, Italy

8 Ospedale Apuano ESTAV Nord Ovest, Massa Carrara, Italy

9 Department of Diagnostic Medicine and Prevention, Radiology Unit, S.Orsola Malpighi, University of Bologna, Bologna, Italy

10 Ospedale Policlinico San Martino, Genova, Italy

11 Diagnostic Imaging and Interventional Radiology, University Hospital of Rome Tor Vergata, Rome, Italy 
therapy, showing promising results (28 mortality day, heparin users vs nonusers $[40.0 \%$ vs $64.2 \%, P=0.029]$, respectively) [3]. A heparin regimen has been also suggested by International Society of Thrombosis and Haemostasis (ISTH) guidance [4]. Moreover, Covid-19induced thrombocytopenia has been postulated as a direct or a sepsis-induced effect, increasing risk of bleeding. Between 23 March and 26 April, we referred 38 Covid-19positive patients (median age $67.4 \pm 12.9 ; 21$ males [68.0\%]) in 11 different Covid-19 hospitals in Italy, with a major bleeding receiving heparin at therapeutic dosages. Bleeding (single: 26/38 [68.4\%]; multifocal 12/28 [42.8\%]) was observed (admitted to the ICU (27/38 [71\%]) that required emergency lifesaving embolization in an interventional radiology suite. The decision to proceed with the embolization rather than suspending heparin administration was weighted according to the patient's clinical condition. In 25/38 (65.7\%) critically unwell patients who were judged to be higher the risk of Covid-19-induced DIC, it was believed unsafe to stop the heparin and endovascular embolization was performed. In 13/38 (34.2\%) less compromised patients, it was deemed safe to stop heparin and embolization was performed in $6 / 13(46.2 \%)$. In the remaining patients, a watch-and-wait strategy was observed. The majority of bleeding was intramuscular (34/ 38 [89.4\%]), 1/38 (2.6\%) cerebral arteriovenous malformation, $1 / 32(2.6 \%)$ in the retroperitoneal space and $1 / 32$ $(2.6 \%)$ splenic. The occurrence of bleeding in this situation has several implications:

- Usually heparin-related bleeding resolves spontaneously at the suspension of the anticoagulation regimen. However, heparin is considered an integral part of Covid-19 treatment and essential in critically ill patients at the risk of DIC.

- A 24/7 fully operational angiographic suite (including angiographic equipment, physician, nurse and technician) should be mandatory in all highly affected Covid Hospitals.

- Bleeding patients usually require blood transfusion which is in short supply during the Covid pandemic due to the lockdown and fear of entering medical facilities

- Biocontainment transfer of Covid-19-positive patients within the hospital is complex and not easy to achieve [5]

In view of these considerations, interventional radiology plays a crucial role and a dedicated treatment pathway should be set up. In this context, a dedicated Covid-19 Hub-Spoke system should be set up with a well-defined protocol for transport and case acceptance in order to avoid hospital spread and resource waste. In conclusion, although major bleeding seems to be rare in Covid-19 patients, its management is complex for several reasons: the need to continue anticoagulation, shortage of blood products, multiple sites of haemorrhage and logistic issues.

Acknowledgments We thank doctor Graham Munneke for the revision of the manuscript.

Funding This study was not supported by any funding.

\section{Compliance with Ethical Standards}

Conflict of interest The authors declare that they have no conflict of interest.

Ethical Approval All procedures performed in studies involving human participants were in accordance with the ethical standards of the institutional and/or national research committee and with the 1964 Helsinki Declaration and its later amendments or comparable ethical standards.

Institutional Review Board (IRB) Approved the letter.

Informed Consent Informed consent was obtained from all individual participants included in the study.

Consent for Publication Consent for publication was obtained for every individual person's data included in the study

\section{References}

1. Dong E, Du H, Gardner L. An interactive web-based dashboard to track COVID-19 in real time. Lancet. 2020;20(5):533-534. https:// doi.org/10.1016/S1473-3099(20)30120-1.

2. Chen $\mathrm{T}, \mathrm{Wu} \mathrm{D}, \mathrm{Chen} \mathrm{H}$, et al. Clinical characteristics of 113 deceased patients with coronavirus disease 2019: retrospective study. BMJ. 2020;368:1091.

3. Tang N, Bai H, Chen X, Gong J, Li D, Sun Z. Anticoagulant treatment is associated with decreased mortality in severe coronavirus disease 2019 patients with coagulopathy. J Thromb Haemost. 2020;18(5):1094-9.

4. Thachil J, Tang N, Gando S, et al. ISTH interim guidance on recognition and management of coagulopathy in COVID-19. J Thromb Haemost. 2020. https://doi.org/10.1111/jth.14860.

5. Liew MF, Siow WT, Yau YW, See KC. Safe patient transport for COVID-19. Crit Care (Lond, Engl). 2020;24(1):94.

Publisher's Note Springer Nature remains neutral with regard to jurisdictional claims in published maps and institutional affiliations. 